\title{
Spatial separation of small and large grains in the transitional disk around the young star IRS $48^{\star}$
}

\author{
V. C. Geers ${ }^{1}$, K. M. Pontoppidan ${ }^{2}$, E. F. van Dishoeck ${ }^{1}$, C. P. Dullemond ${ }^{3}$, \\ J.-C. Augereau ${ }^{4}$, B. Merín ${ }^{1,5}$, I. Oliveira ${ }^{1}$, and J. W. Pel ${ }^{6}$ \\ 1 Leiden Observatory, PO Box 9513, 2300 RA Leiden, The Netherlands \\ e-mail: vcgeers@strw.leidenuniv.nl \\ 2 Hubble Fellow, Division of GPS, Mail Code 150-21, California Institute of Technology, Pasadena, CA 91125, USA \\ 3 Max-Plank-Institut für Astronomie, Koenigstuhl 17, 69117 Heidelberg, Germany \\ ${ }^{4}$ Laboratoire d'Astrophysique de l'Observatoire de Grenoble, BP 53, 38041 Grenoble Cedex 9, France \\ 5 Research and Scientific Support Department (ESTEC/ESA), Keplerlaan 1, 2200 AG Noordwijk, The Netherlands \\ ${ }^{6}$ Kapteyn Astronomical Institute, Landleven 12, 9747 AD Groningen, The Netherlands
}

Received 22 March 2007 / Accepted 28 April 2007

\begin{abstract}
Aims. We present spatially resolved mid-infrared images of the disk surrounding the young star IRS 48 in the Ophiuchus cloud complex. The disk exhibits a ring-like structure at $18.7 \mu \mathrm{m}$, and is dominated by very strong emission from polycyclic aromatic hydrocarbons at shorter wavelengths. This allows a detailed study of the relative distributions of small and large dust grains.

Methods. Images of IRS 48 in 5 mid-infrared bands from 8.6 to $18.7 \mu \mathrm{m}$ as well as a low resolution N-band spectrum are obtained with VLT-VISIR. Optical spectroscopy is used to determine the spectral type of the central star and to measure the strength of the $\mathrm{H} \alpha$ line.

Results. The $18.7 \mu \mathrm{m}$ ring peaks at a diameter of $110 \mathrm{AU}$, with a gap of $\sim 60 \mathrm{AU}$. The shape of the ring is consistent with an inclination of $i=48^{\circ} \pm 8^{\circ}$. In contrast, the 7.5-13 $\mu \mathrm{m}$ PAH emission bands are centered on the source and appear to fill the gap within the ring. The measured PAH line strengths are 10-100× stronger than those typically measured for young M0 stars and can only be explained with a high PAH abundance and/or strong excess optical/UV emission. The morphology of the images, combined with the absence of a silicate emission feature, imply that the inner disk has been cleared of micron-sized dust but with a significant population of PAHs remaining. We argue that the gap can be due to grain growth and settling or to clearing by an unseen planetary or low-mass companion. IRS 48 may represent a short-lived transitional phase from a classical to a weak-line T Tauri star.
\end{abstract}

Key words. stars: pre-main sequence - stars: planetary systems: protoplanetary disks - stars: circumstellar matter - astrochemistry stars: individual: IRS 48

\section{Introduction}

The number of circumstellar dust disks detected around young stars has increased dramatically over the last decades thanks to a variety of ground- and space-based observations. Studies of the Spectral Energy Distributions (SEDs) of young stellar objects at various ages indicate how these disks evolve from optically thick, massive gas-rich disks to the more optically thin, tenuous gas-poor disks by a combination of gas accretion, grain growth, planet formation and photo-evaporation of the gas.

Only very few spatially resolved mid-infrared images have been presented. Some tenuous disks around 5-15 Myr stars show evidence for gap formation and spiral arm structures (e.g. Jayawardhana et al. 1998; Augereau et al. 1999; Liu 2004), and this has been interpreted as evidence for the presence of forming planets clearing out a ring of dust and gas. For younger disks around $\sim 1$ Myr old stars, there is still very little direct evidence for the formation of gaps (Fujiwara et al. 2006).

* Based on observations obtained at the European Southern Observatory, Paranal, Chile, within the observing program 075.C-0211, and observations obtained with the WHT operated on the island of La Palma by the Isaac Newton Group.
An obvious step toward the formation of planetesimals in disks is the coagulation and growth of the sub-micron sized grains accreted from the proto-stellar envelope (Dominik et al. 2007). Although the end results are plainly visible in our own planetary system, as well as in the emerging wealth of exosolar planets, the details and mechanisms of dust evolution in proto-planetary disks are not well understood. Very strong grain growth may lower the dust opacity enough to form an apparent gap in the disk at mid-infrared wavelengths, similar to those attributed to dust clearing by planets (D'Alessio et al. 2005; Tanaka et al. 2005). A wide range of recent observational results indeed suggest that significant grain growth is a common occurence in disks (e.g. van Boekel et al. 2005; Kessler-Silacci et al. 2006; Natta et al. 2007).

However, some observations complicate this picture. A subset of protoplanetary disks shows strong emission features from extremely small grains - Polycyclic Aromatic Hydrocarbons (PAHs) (Acke \& van den Ancker 2004; Geers et al. 2006), even in disks with apparent gaps. How do these disks fit into the general picture of grain evolution? Are they evidence of grain size segregation, leaving only the small grains in the upper layers of the disk? Is gas still present in these regions? 

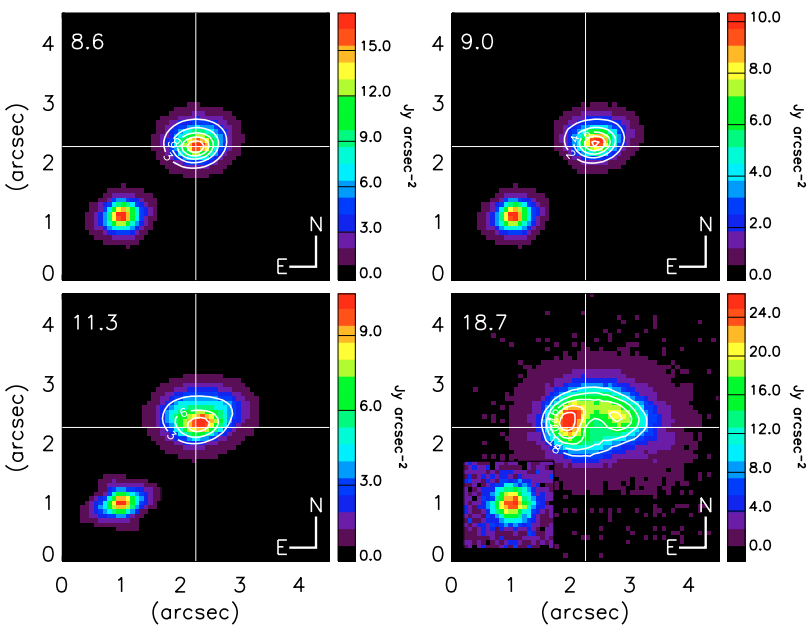

Fig. 1. VISIR images of IRS 48, with PSF standard shown in inserts; the crosshair indicates the center of the emission at $8.6 \mu \mathrm{m}$. All images up to $12 \mu \mathrm{m}$ are dominated by PAH emission. The $11.9 \mu \mathrm{m}$ image (not shown) is similar to that at $11.3 \mu \mathrm{m}$. The contours are indicated for 3,6 , 9, 12, $15(8.6 \mu \mathrm{m}), 2,4,6,8,10(9.0 \mu \mathrm{m}), 3,6,9,12(11.3 \mu \mathrm{m})$ and 8 , $12,16,20,24(18.7 \mu \mathrm{m}) \mathrm{Jy} \operatorname{arcsec}^{-2}$.

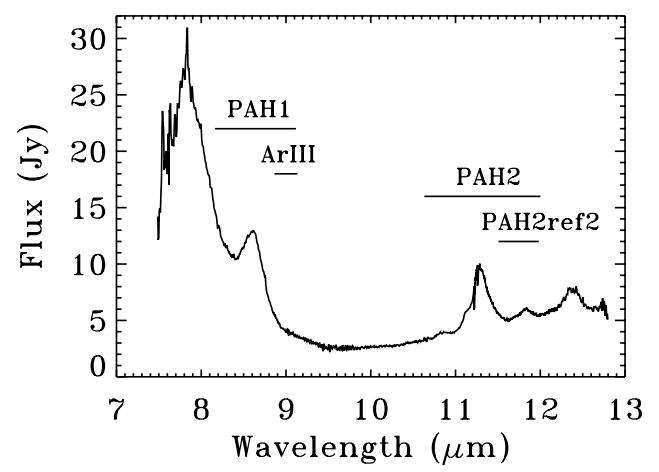

Fig. 2. VLT-VISIR N-band spectrum of IRS 48. Spectral width of transmission curves of VISIR image filters are indicated.

In this letter, we present one of the first spatially resolved mid-infrared images and spectroscopy of a disk around a young (few Myr) star with a rising mid-IR SED, IRS 48 in Ophiuchus (Wilking et al. 1989) (catalogued as WLY 2-48, 1627 37.19, $-243035.0 \mathrm{~J} 2000$ ), which is no longer surrounded by an envelope. This source shows exceptionally strong PAH emission at 3.3 and $7.7-12.3 \mu \mathrm{m}$ in the inner part of the disk as well as an apparent inner gap seen at $18.7 \mu \mathrm{m}$. We will discuss the origin of the $18.7 \mu \mathrm{m}$ gap in the context of the PAH images which show that the gap is not cleared of material.

\section{Observations of IRS 48 and data reduction}

Images were obtained with VISIR on the Very Large Telescope in 5 mid-infrared bands at 8.6, 9.0, 11.3, 11.9 and $18.7 \mu \mathrm{m}$ on June 9, 2005 (Fig. 1). VISIR N-band spectroscopy was taken on June 12, 2005, in the low resolution settings at 8.8, 9.8, 11.4 and $12.2 \mu \mathrm{m}$, with a typical resolving power of $\lambda / \Delta \lambda \sim 300$ (Fig. 2). The telescope was operated using a standard chop-nod scheme with chop-throws of $10^{\prime \prime}$. The data were reduced using a combination of the ESO pipeline (v. 1.3.7) and our own IDL routines.

A WYFFOS optical spectrum was obtained at the William Herschel Telescope (WHT) on La Palma on May 4, 2006, with a resolving power of $R \sim 1600$ (Fig. 3). It indicates a M0 spectral

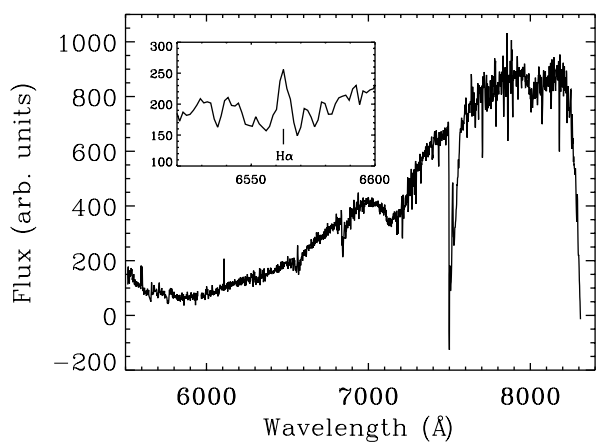

Fig. 3. WHT-WYFFOS optical spectrum of IRS 48. The inset shows a blow-up of the $\mathrm{H} \alpha$ line.

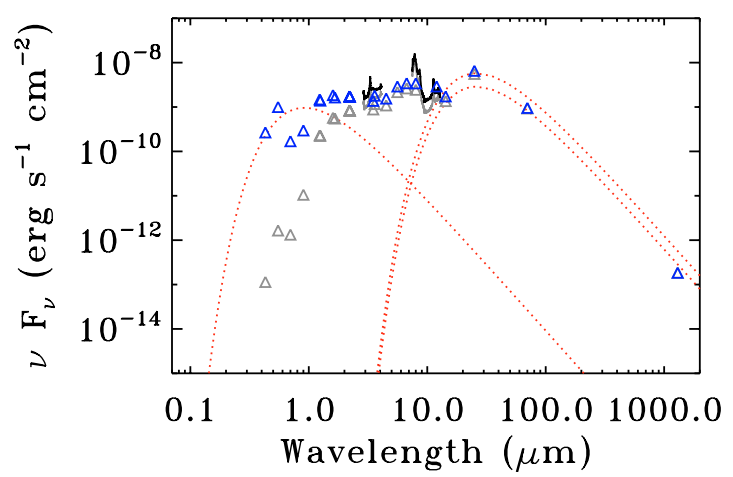

Fig. 4. The SED of IRS 48 based on the photometry listed in Sect. 2. Grey triangles are literature photometry, blue triangles are dereddened, with $A_{\mathrm{v}}=7$; black solid lines are ISAAC L-band and VISIR N-band spectra; orange dotted lines show 3 blackbodies, at $T_{\text {eff }}=4000 \mathrm{~K}$ $\left(0.63 L_{\odot}\right)$ and $140 \mathrm{~K}$, the latter scaled to 1.9 and $3.8 L_{\odot}$.

type $\left(T_{\text {eff }}=3800 \mathrm{~K}\right)$ with an error of less than 2 spectral subtypes. $A_{v}=7 \pm 1 \mathrm{mag}$ is derived from the optical spectrum as in Oliveira et al. (in prep.). $\mathrm{H} \alpha$ is detected with an Equivalent Width (EW) of $5.9 \AA$, which classifies IRS 48 as a weak-line T Tauri star. Our derived spectral type is much later than that of Luhman \& Rieke (1999), who found it to be earlier than F3 based on a K-band spectrum (taken sometime between July 1994 and June 1996). This discrepancy remains to be understood and will be discussed in Sect. 4.2.

Photometry used to construct the SED (Fig. 4) includes NOMAD (Zacharias et al. 2004), USNO-B (Monet et al. 2003), 2MASS (Skrutskie et al. 2006), ISOCAM (Bontemps et al. 2001), Spitzer Space Telescope IRAC and MIPS (3.6, 4.5, 5.8, $8.0,24,70 \mu \mathrm{m}$ ) from the c2d legacy program database (Evans et al. 2003) and IRAM $1.3 \mathrm{~mm}$ (André \& Montmerle 1994).

\section{Results}

The circumstellar disk is spatially resolved in all 5 VISIR images, shown in Fig. 1. An extent of 1.2-1.9" is derived along the semi-major axis, corresponding to 150-230 AU diameter; the actual extent increases with wavelength. Ducourant et al. (2005) measured a proper motion consistent with the surrounding Oph sources placing IRS 48 firmly at a distance of 125 pc (de Geus et al. 1989). All 5 bands are resolved with respect to the standard star PSF along the east-west direction, showing elongated surface brightness profiles consistent with a circumstellar disk. We derive an inclination of $i=48^{\circ} \pm 8^{\circ}$ from the semi-major and minor axes of ellipsoidal contours fitted to the $18.7 \mu \mathrm{m}$ image, and a position angle of $98 \pm 3^{\circ}$ East of North. 
The most striking result is that the $18.7 \mu \mathrm{m}$ Q-band image shows an asymmetry in the surface brightness and a gap in the center. We interpret the apparent shape as being due to an inclined ring-shaped disk. No point source is seen within this gap. Its diameter as measured from the inner edges of the ring along the semi-major axis equals $0.5^{\prime \prime}$ or $\sim 60 \mathrm{AU}$, corresponding to a gap with a radius of $\sim 30 \mathrm{AU}$. In contrast, the PAH emission at 8.6 and $11.3 \mu \mathrm{m}$ is centrally peaked with resolved wings beyond a point-source, and it appears to originate from the apparent gap in the Q2 image. The center of the images shifts by $\sim 0.2^{\prime \prime}$, likely due to pointing error, as indicated by similar shifts in the standard star positions. The PAH off-band filters at 9.0 and $11.9 \mu \mathrm{m}$ both resemble the 8.6 and $11.3 \mu \mathrm{m}$ images respectively. Given the strength of the PAHs (see Fig. 2), it is assumed that both off-band filters also probe PAH emission or very small grains.

The VISIR N-band spectrum (Fig. 2) shows clear PAH features at $8.6,10.8,11.3,11.9$ and $12.5 \mu \mathrm{m}$. Our $11.3 \mu \mathrm{m}$ line flux is $2.5 \times 10^{-14} \mathrm{~W} \mathrm{~m}^{-2}$. The PAH features around this M0 star are as strong as the strongest features observed around Herbig Ae/Be stars (Acke et al. 2004), and make it the latest type young star with detected PAHs. No silicate emission feature at $9.7 \mu \mathrm{m}$ is detected. A VLT-ISAAC L-band $(2.8-4.2 \mu \mathrm{m})$ spectrum, obtained as part of the ice survey by van Dishoeck et al. (2003), taken on 2002, May 5 (sample and reduction described in Pontoppidan et al. 2003), shows the presence of a strong $3.3 \mu \mathrm{m}$ $\mathrm{PAH}$ feature and the $4.05 \mu \mathrm{m} \mathrm{Br} \alpha$ line (included in Fig. 4).

\section{Discussion}

\subsection{Gap in the disk}

Gaps in disks around T Tauri stars have been inferred for a few sources (Calvet et al. 2005; Sicilia-Aguilar et al. 2006; Brown et al. submitted) but entirely on the basis of the SED, not spatially resolved images such as presented here. The $18.7 \mu \mathrm{m}$ image suggests a gap with a radius of $30 \mathrm{AU}$ in the dust disk. However, the PAH-band images show that this gap cannot be entirely devoid of small particles. The near-infrared excess at $1-3 \mu \mathrm{m}$ also suggests that some hot dust still exists in a small ring of material or a puffed-up inner rim in the inner few AU, i.e., the disk shows a gap and not a hole. Interestingly, the SED of IRS 48 (Fig. 4) does not reveal the presence of a gap, possibly due to masking of the dip at $\sim 5-15 \mu \mathrm{m}$ by the strong PAH features and other types of very small grains (VSGs) present in the gap. Quantum-heated PAHs and very small grains reach a much higher average temperature than thermal grains, radiating strongly in distinct PAH features and continuum at $5-15 \mu \mathrm{m}$, while being weaker at $20 \mu \mathrm{m}$.

Gaps in disks have been interpreted in the context of photoevaporation of gas and small dust grains by the central star. This mechanism is excluded here since it should create a similar gap in the PAH filter images. Another way to make an apparent gap is to lower the dust opacity by grain growth, effectively removing the silicate dust particles responsible for the continuum emission between a few and $\sim 15 \mu \mathrm{m}$. Theoretical models show that grain growth occurs on short time scales, with the shortest growth times in the inner parts of the disk (Weidenschilling 1997; Dullemond \& Dominik 2005). Furthermore, larger grains settle to the mid-plane faster than smaller grains, creating a strong size segregation in the vertical direction, which would explain the strong feature/continuum ratio of the PAH features (Dullemond et al., subm.). The lack of a significant silicate feature at $9.7 \mu \mathrm{m}$ supports the idea that in the inner disk most of the dust is in larger micron-sized grains. However, this scenario has difficulties explaining the presence of a sharply defined ring structure.

A third way of gap formation is by the clearing out of gas and dust by a planet forming inside the disk (e.g. Klahr \& Lin 2001) or a low-mass companion. Planets are expected to clear out larger $(>100 \mu \mathrm{m})$ grains much more rapidly than gas and small particles by tidal interaction and can cause sharp edges in images (Paardekooper \& Mellema 2004; Quillen et al. 2004). Particularly, Rice et al. (2006) predict that larger dust grains are filtered out at the outer edge, presumably leading to a build-up of large dust grains and a clearly defined ring of material at the outer edge, while smaller grains (e.g. PAHs) continue to accrete inwards along with the gas, which would explain the continued presence of PAH emission in the gap. Interferometric observations at millimeter wavelengths to constrain the distribution of even larger (mm-sized) particles can further test these scenarios.

\subsection{Source of central luminosity}

The SED of IRS 48 is peculiar for several reasons. It shows a very strong bump at $25 \mu \mathrm{m}$ which appears to be consistent with a single temperature blackbody of $\sim 140 \mathrm{~K}$. This emission bump includes $18 \mu \mathrm{m}$ and should be associated with the "ring" seen in the Q2-band image. The apparent luminosity from the $25 \mu \mathrm{m}$ bump is $\sim 1.9-3.8 L_{\odot}$. If this ring is assumed to be the sole source of the bump and if we assume the ring to be an optically thick annulus with a covering fraction of at most 0.2 with respect to the star, it follows that the central source should have a luminosity of at least $10-20 L_{\odot}$, which is more than 20 times stronger than expected for a 1 Myr M0 star.

External heating by nearby bright sources can be excluded from our own VISIR, Spitzer IRAC and ESO archive NACO images, as well as recent multiplicity surveys (Haisch et al. 2004). The possibility of a close ( $<175 \mathrm{AU})$ binary with a higher mass early type star can be excluded for lack of spectral features associated with early type stars in our optical spectrum.

The most likely scenario is the presence of excess UV emission, which is absorbed by a local foreground layer of material, such as a strongly inclined disk with an inner rim that is puffed up due to temporary accretion events, or absorption by the flared outer disk. A fully edge-on disk is considered unlikely; it could produce the observed SED, but it would be inconsistent with the Q2-band image.

One example of excess luminosity is through accretion. This would require an accretion luminosity much larger than the star itself. No X-ray emission has been detected toward this object by Chandra nor XMM-Newton (Grosso, priv. comm.) and both $\mathrm{H} \alpha$ and $\operatorname{Pf} \beta$ are relatively weak, which argues against strong accretion.

An alternative explanation might be that this disk has recently undergone a FU Orionis outburst. During a major FU Ori-type accretion event, the temperature of the disk increases over a span of 1-10 years from a few hundred to a few thousand $\mathrm{K}$, and will dominate the spectrum even for optical wavelengths (Hartmann 2001). The apparent spectral type can change by several types, from late K-M to F-G type. This may explain the discrepancy between the $<\mathrm{F} 3$ spectral type determination observed between 1994 and 1996 and the M0 type determination in 2006. Heating of the disk would help the PAHs in two ways: any PAHs trapped in ices would be evaporated boosting the PAH abundance and the PAHs could be thermally excited. 


\subsection{PAH feature strength}

IRS 48 is exceptional in its very prominent PAH features, both in strength and feature-over-continuum ratios, 100-1000 times stronger than recent model predictions for M0 stars (Geers et al. 2006). Comparing our measured $11.3 \mu \mathrm{m}$ line strength with their Fig. 9, we find that it would be consistent with the radiation field of a $\sim 6000 \mathrm{~K}$ central star, in a disk model with a PAH abundance of $5 \times 10^{-5}$ with respect to hydrogen, typical of the general interstellar medium but higher than inferred for disks. Assuming this abundance, the strong PAH features would be consistent with excess optical/UV luminosity at a level corresponding to several hundred times the average interstellar radiation field at a radius of $100 \mathrm{AU}$, which is consistent with recent optical/UV strengths of T Tauri stars inferred by Bergin et al. (2003).

The case of IRS 48 suggests that the combination of high feature-over-continuum PAH bands and the absence of silicate features together with a rising SED at $\lambda>12 \mu \mathrm{m}$ is also an indicator for the presence of gap formation through grain growth, which is not revealed in broadband SEDs. Mid-infrared spectroscopy and high-spatial resolution narrow-band imaging with 8-m class telescopes, combined with millimeter interferometric imaging, of a much larger sample of objects is crucial to determine whether IRS 48 is just a peculiar object or whether it forms part of a new class of transitional disks.

Acknowledgements. We thank A. Smette for crucial help in obtaining the VISIR data and N. Grosso for providing X-ray information. KMP is supported by NASA through Hubble Fellowship grant 01201.01 awarded by the STScI, which is operated by the AURA, for NASA, under contract NAS 5-26555. Astrochemistry in Leiden is supported by a Spinoza grant from The Netherlands Organization for Scientific Research (NWO).

\section{References}

Acke, B., \& van den Ancker, M. E. 2004, A\&A, 426, 151 André, P., \& Montmerle, T. 1994, ApJ, 420, 837
Augereau, J. C., Lagrange, A. M., Mouillet, D., \& Ménard, F. 1999, A\&A, 350, L51

Bergin, E., Calvet, N., D’Alessio, P., \& Herczeg, G. J. 2003, ApJ, 591, L159

Bontemps, S., André, P., Kaas, A. A., et al. 2001, A\&A, 372, 173

Calvet, N., D’Alessio, P., Watson, D. M., et al. 2005, ApJ, 630, L185

D’Alessio, P., Hartmann, L., Calvet, N., et al. 2005, ApJ, 621, 461

de Geus, E. J., de Zeeuw, P. T., \& Lub, J. 1989, A\&A, 216, 44

Dominik, C., Blum, J., Cuzzi, J. N., \& Wurm, G. 2007, in Protostars and Planets V, University of Arizona Press, ed. B. Reipurth, D. Jewitt, \& K. Keil, 783

Ducourant, C., Teixeira, R., Périé, J. P., et al. 2005, A\&A, 438, 769

Dullemond, C. P., \& Dominik, C. 2005, A\&A, 434, 971

Evans, N. J., Allen, L. E., Blake, G. A., et al. 2003, PASP, 115, 965

Fujiwara, H., Honda, M., Kataza, H., et al. 2006, ApJ, 644, L133

Geers, V. C., Augereau, J.-C., Pontoppidan, K. M., et al. 2006, A\&A, 459, 545

Haisch, Jr., K. E., Greene, T. P., Barsony, M., \& Stahler, S. W. 2004, AJ, 127, 1747

Hartmann, L. 2001, Accretion Processes in Star Formation (Cambridge University Press)

Jayawardhana, R., Fisher, S., Hartmann, L., et al. 1998, ApJ, 503, L79

Kessler-Silacci, J., Augereau, J.-C., Dullemond, C. P., et al. 2006, ApJ, 639, 275

Klahr, H. H., \& Lin, D. N. C. 2001, ApJ, 554, 1095

Liu, M. C. 2004, Science, 305, 1442

Luhman, K. L., \& Rieke, G. H. 1999, ApJ, 525, 440

Monet, D. G., Levine, S. E., Canzian, B., et al. 2003, AJ, 125, 984

Natta, A., Testi, L., Calvet, N., et al. 2007, in Protostars and Planets V, University of Arizona Press, ed. B. Reipurth, D. Jewitt, \& K. Keil, 767

Paardekooper, S.-J., \& Mellema, G. 2004, A\&A, 425, L9

Pontoppidan, K. M., Fraser, H. J., Dartois, E., et al. 2003, A\&A, 408, 981

Quillen, A. C., Blackman, E. G., Frank, A., \& Varnière, P. 2004, ApJ, 612, L137

Rice, W. K. M., Armitage, P. J., Wood, K., \& Lodato, G. 2006, MNRAS, 373, 1619

Sicilia-Aguilar, A., Hartmann, L., Calvet, N., et al. 2006, ApJ, 638, 897

Skrutskie, M. F., Cutri, R. M., Stiening, R., et al. 2006, AJ, 131, 1163

Tanaka, H., Himeno, Y., \& Ida, S. 2005, ApJ, 625, 414

van Boekel, R., Min, M., Waters, L. B. F. M., et al. 2005, A\&A, 437, 189

van Dishoeck, E. F., Dartois, E., Pontoppidan, K. M., et al. 2003, The Messenger, 113,49

Weidenschilling, S. J. 1997, Icarus, 127, 290

Wilking, B. A., Lada, C. J., \& Young, E. T. 1989, ApJ, 340, 823

Zacharias, N., Monet, D. G., Levine, S. E., et al. 2004, Bull. Amer. Astron. Soc., 1418 